the swelling of the skin near it were more marked. On this occasion the swelling and pain were not so severe as during the first attack. This time the whole course was febrile like the first one. In other respects there was no difference noticed. This time there was no external wound on any part of the body, the thumb haring quite healed up. Before any internal medicine was given I had an opportunity of examining the urine and the same reaction was obtained. On this occasion there was a slight trace of albumin and it was found that saturating with neutral ammonium sulphate produced a dirty grey amorphous deposit. This was filtered, and the filtrate gave the reaction rather feebly, while the precipitate, although washed repeatedly with saturated neutral ammonium sulphate solution, gave the reaction strongly. However, on washing another sample with distilled water the reaction-producing substance was removed frrm the precipitate.

Here I may state that the presence of this substance is likely to mislead one as regards the presence of peptone. Fehling's solution produces a beautiful pale rosy colour identical with that of peptone reaction. The absence of peptone in this case was proved by the salicyl sulphonic acid and neutral ammonium sulphate test, while a control test with peptone added to urine failed to give the purple reaction with an alkali.

Now, finally, I have to say a few words regarding the nature of this substance. One thing is certain, that this reaction is not found in any other sample of urine which $I$ have examined. However, I have reason to believe that the substance from which this pigment is produced does exist in a modified form in the urine in certain cases. In the sample of urine treated with bydrochloric acid mentioned above I have said that I was able to obtain the reaction by adding caustic soda. However, in my first experiment, there being probably too much brown deposit used, I found that the alkaline solution was brownish in colour, so I added a little hydrogen peroxide with a view to see if the brownish pigment could be so altered as to reproduce the pink reaction. This surmise was at once verifed, and since then I have tried the same test with two other samples in which the pigment was not present in fresh urine and the brownish deposit had assumed a pale rosy colour similar to that which is produced by leaving the purple deposit in contact with hydrogen peroxide for a long time. This was in a case of scurvy and the other one was in a case of fever brought on by slight hepatic congestion. In other cases I have not obtained this modified reaction, and it is interesting to note that the urine of the patient whose case gave this reaction gives neither the original nor the modified one now that she has quite recovered.

I may state here that the patient is a martyr to asthma; yet during a rather severe fit I have failed to obtain the reaction with her urine. In the mixture given for this illness will be found tinctura chloroformi composita. Now thi contains carmine as a colouring agent; so my attention was directed to prove whether or not this reaction was due to some special idiosyncrasy on the part of the patient to excrete this substance. Experiments on other people failed to give the reaction. Every single drug was tried separately and collectively on myself with negative result. On the other hand carmine was added in minute amount to healthy urine and the reaction was at once obtained; however, this reaction is soon destroyed by hydrogen peroxide. To be sure that carmine had nothing to do with the urinary reaction I availed myself of the opportunity afforded by the second attack, when the drug was withheld, yet there was no difference in the reaction produced. The blood was examined for the reaction during the second attack with negative result. At the suggestion of Dr. Lyons I tried Bremer's test with Congo-red, methylene-blue, methylene-blue and eosin fuchsin, and erythrosin. There was no difference noted in the staining reaction except in the case of Congo-red. This stained the blood film more deeply than in a control case, quite the reverse of that which happens in diabetic blood. I have not had an opportunity of verifying this observation under more "rigid conditions, so I cannot say whether or not there is a specific staining reaction present in this disease. The filtered pigment being allowed to dry in air becomes bluish in colour. As regards the nature of this disease I abstain from making any observations now. In conclusion, I have to state that from a record of 195 cases of urine analysis $I$ have not seen a single case giving this reaction.

Bombay.

\section{ECZEMA AND THE ALLIED DISEASES: AN OUTLINE OF THEIR ETIOLOGY, PATHOLOGY, AND TREATMENT. ${ }^{1}$}

BY W. T. FREEMAN, M.D. DuRH., F.R.C.S. ENG., SURGEOX IN CHARGE OF THE SKIY DEPARTMEXT A' THE REAIHSi DISPEXSARY.

I HAVE been asked to open a discussion on eczema ard the allied diseases with the hope that a large number of members will express their opinions regarding the etiology, pathology, and treatment of this widely.known and interesting group of disorders.

First of all, how are we to define eczema? On this very subject at the Edinburgh meeting of the British Medical Association in 1898 an eminent dermatologist, quoting Jobn Hunter, said "that definitions were the most damnable things," and they are so. I would define eczema as "a serous inflammation of the skin not produced by any form of external irritants." At some stage or other of its evolution there is or has been exudation of serum. In the acute stage, at all events, the clinical signs of inflammation-heat, pain, redness, and swelling -are all present, but the rise of body temperature that accompanies other forms of inflammation is almost invariably absent. I agree with those authorities who regard an erythematous, a papular, a vesicular, a pustular, a dry or a scaly eczema, as simply stages of the same complaint. I would recognise as the great sub-variety of eczema "eczema seborrhoicum," and one means by that an eczema occurring in a patient who also suffers from seborrhoea, and it usually commences and spreads after the same fashion as seborrhœe, starting on the scalp and progressing slowly downwards over the body and limbs. Sweat eczema, follicular eczema, or sun eczema barely deserve the name of sub-varieties, and eczema marginatum is body ringworm attacking sweaty parts of the skin.

That there is real difficulty and confusion in connezion with the terminology of this disease is very easily exemplified, for do we not often hear people talk of the secondary eczema produced by the irritation of the itch parasite? Now this does not fit in with my definition of the complaint nor with any of the terminology usually accepted by dermatologists. Scabies, in fact, is produced by a direct irritant to the skin, and the various skin conditions associated with it should be varieties of dermatitis. If we are to retain the old familiar term-and it would certainly take a lot of knocking out-we should at all events have a clear image in our minds of what we mean by eczema and what we mean by dermatitis. We all appreciate one great difference between a dermatitis and eczema. The former is under control-i.e, the irritant can under ordinary circumstances be removed; but it is not $\$ 0$ with the latter disease, only too frequently does it baftle us and get out of control.

\section{The Etrology of Eczena.}

Personally I prefer to consider eczema as a distinct disease. as an entity as clearly defined from other diseases as is, for instance, enteric fever from pneumonia. Hereby I commit myself to the parasitic theory, believing that it is brought about by the behaviour of a specific organism, possibly the "morococcus" of Unna, just as the other compared diseases are brought about by the doings of the bacillus of typhoid fever or the pneumococcus of inflammation of the lung. When we speak of eczema being produced, as it very often is, by a chill, or through nervous influence, or by a gouty inheritance, or by diabetes, we do not mean that these conditions are the actual cause of it. We mean that they are the exciting causes, that they reduce the soil suddenly, or perhaps quietly, to a condition favourably receptive to the eczema "morococcus." A chill may equally be the excitir: cause of eczema, pneumonia, scarlet fever, or rheumatic fever, or of any other complaint, and the public at all events are quite satisfied with this sole explanation. Fiven to us. knowing more of the specific cause in each case, there is still a veiled pathological dreamland; for why should a chill in one instance favour the attack of the eczema organism, ard 1 A paper read before the Pathological Society of Reading on
June 14th, 1900. 
in another that of the pneumococcus, and in another that of the tubercle bacillus? Well, there is the individual to be considered; the altered conditions produced by the chill in the one case may be very different to the conditions brought about by it in the other. Then comes, too, the question of the "survical of the fittest"-I mean of the fittest organism. One particular set of bacilli in the neighbourhood of the depressed patient may be infinitely more numerous, or, again, one bacillus may be healtby and fit for attack while another may itself be suffering from a chill of some kind or be knocked ont at the time from the attack of a better conditioned organism. We must not confuse the attentions of the staphylococcus pyogenes aureus or albus with those of a specific organism. Their doings are accidental and prerentable.

In the January and February numbers for 1899 of the Dermatological Journal Dr. Leslie Roberts has a most excellent and exhaustive article dealing with the pathology of eczema. He opens it by drawing attention to the inflammatory, secretory, excretory, and digestive function of the epithelium of the skin, or rather of the cellular components of the epithelial layer. To think of special skin cells possessing an inflammatory function is only funny upon frst consideration, for, after all, they are strictly comparable to the cells of the cpithelial layer along the alimentary and other tracts. There is no doubt, so far as our present knowledge goes, that only individual sets of cells along the mucous tracts do individual business, and it is fair analogy to give separate functions to separate skin cellular tracts. As to the digestive functions of the skin Dr. Roberts clearly proves them by actual test-tube reactions carefully watched in an incubator. But in the more practical part of his paper he allows no sharp line to be drawn between dermatitis and eczema. There are three main propositions that I would ask the members of this society to express their opinions upon. 1. Is eczema an entity-a specific disease? 2. Is it due to the fact that certain functional epithelial cells are excited into over activity by some kind of irritant? 3. Is there any distinction at all to be drawn between eczema and dermatitis?

I will now cite just a few practical examples of the dificulty of the question. At a meeting of the Dermatological Society of London on March 14th I examined a patient brought forward as a case of "pyodermia." At a meeting of the British Dermatological Society on Feb. 23th I examined some pure cultivations of the staphylococcus aureus from a case of " acute recurring eczema." Then, again, there are the cases of exfoliative dermatitis which nowadays we frequently see, and which no doubt we saw quite as frequently in the days gone by, but we simply did not bother to differentiate and called them all "eczema." The sceptic will say that it did not matterthe patients did just as well and what particular coccus was knocking about was quite immaterial. This, however, is not true, for without a true pathological and bacteriological idea of what is going on it is quite impossible to methodically treat any case of skin disease-or for the matter of that any kind of disease whatever.

Some of the conditions simulating eczema are not easy of diagnosis. To compare dry eczema with psoriasis, for instance, or seborrhceic dermatitis with psoriasis, or eczema with seborrboic dermatitis, we must of ten simply depend upon the general picture as shown to us. The scales of seborrhoic disease are more or less oily and there is not the red dry surface left as after the removal of the scales of psoriasis or the greater or lesser attempt at exudation as noted in the case of the cleansed eczema patch. Dermatitis herpetiformis is often not recognised by the general physician, but it is very important that it should be. Here again the general picture, and particulariy the grouping of the vesicles and bullæ, will generally settle the question. The early stage, the premycosic stage of granuloma fungoiles, should be recognised. Xot long ago at a meeting of one of the dermatological societies at which I was present a case was brought forward in a man as pityriasis rubra. It was recognised by Dr. H. R Crocker and by others who were present as an early case of mycosis fangoides. There were, however, no signs of tamours present. They developed nevertheless within six months and within twelve months the patient was dead. Inceed, the dicease is so invariably fatal that only the worst rognosis can be given. This man as he was shown for the frst time corresponded with les hommes rouges of the French cicool. These cases also appear in their early stage pretty cten as obstinate general cases of acute or subacute eczema, or, if you wish to call them so, as cases of acute or subacute general dermatitis. Indeed, a very obstinate general eczema should be regarded with grave suspicion. Having once seen one or two cases of developed granuloma fungoides one would not be likely to miss even a single very small tumour. It is useless to remove these tumours.

Patches of eczema and lupus may be very difficult to distinguish the one from the other. I can think now of a man being shown at the Dermatological Society a year or two ago with patches on his hands. They were on the back of the hand, were abruptly circumscribed, were covered with a slight crust, and were thickened. They looked very much like patches of lupus, but I believe that they subsequently turned out to be patches of eczema. There was much difference of opinion upon the condition as then shown. The abrupt margins, the thickening, and the slight crusts made them look very much like lupus. Such cases would have to be watched. If the patches spread by their edges steadily advancing and if some amount of scarring were left behind, the diagnosis would be for lupus, and a tuberculous history might still further assist this diagnosis. Bat if they spread more diffusely and an improvement was brought about by treatment and without any scarring being left behind the such patches could be definitely fixed as those of a chronic eczema.

The subject of seborrhoea and its allied conditions was brought forward at a discussion at which I was present only last month. Mr. W. Anderson, Dr. Stephen Mackenzie, Dr. H. R. Crocker, and others took part in the discussion. To enter into the details would take too long a time, but the close connexion or relationship between pityriasis of the scalp and seborrhoea sicca et oleosa, and seborrhceic dermatitis and alopecia, was clearly brought out. It seemed to be generally agreed that the waxy and oily forms of seborrhoea were the same disease either in different stages or on different soils. The dry scalp pityriasis is possibly a condition by itself. Seborrhœic dermatitis, or eczema seborrhoicum, is probably seborrhoea complicated by the invasion of the staphylococcus pyogenes aureus et albus. All of these conditions lead to baldness of the scalp or of any other part of the body, and it will be remembered that Sabouraud has lately demonstrated a cocoon of micro-bacilli and epithelium which belongs to, and is the destroying agent of, alopecia areata and which is also, he says, the actual cause of seborrhoea. He also says that these micro-bacilli pave the way for the various pus organisms which so frequently complicate these conditions. Just in passing I would say that there is no doubt that some forms of alopecia of the scalp are produced by the microsporon or by the endo-ectothrix fungus of ringworm.

There are one or two other forms of disease allied to eczema to which I ought to allude. They are amongst those produced by pus cocci. I think we may at once brush aside "ectbyma" as merely being an obsolete name for a form of pustular eczema or for impetigo contagiosa attacking such parts as the legs. Impetigo contagiosa we must accept as a separate entity. We are all familiar with the crusty patches of this disease, which appear, indeed, to be simply dabbed on the surface, and which, unless irritated, have no sur. rounding erythema and leave no after scarring. Then there is that particular form of sycosis produced by the staphylococcus pyogenes. I am speaking, of course, only of the simple form of folliculitis known as sycosis and not of the hyphogenic sycosis. It is said that it is more painful and difficult to epilate in the coccogenic than in the byphogenic form, but the true test is the microscope.

I heard it laid down as an axiom the other day by Dr. Radcliffe Crocker that we should not use the terms "impetigo" or "impetiginous eczema" unless we are referring to impetigo contagiosa. Any authoritative statement of this kind is useful as tending to prevent loose descriptions and definitions. Then what do we mean by "prurigo"? I think it is a separate disease. The history almost always dates from infancy, the earlier manifestations being, perbaps, those of lichen urticatus. It attacks by preference the extensor surfaces and the leading symptoms are the hard, dry papules often topped by a crust of blood the result of scratching. There is something about the scratches which is difficult to describe but which is yet almost pathognomonic. The disease lasts indefinitely; the symptoms admit of amelioration bat the condition is not, strictly speaking, curable. I saw an uncommon case of prurigo in a boy the other day in which the face and hands were alone affected.

All the varieties of erythema might be considered allic 
diseases of eczema, as also dermatitis due to pediculi or to the acarus of scabies, or to the handling of the primula obconica; also here in Reading we should not forget the skin irritation caused by the handling of hyacinth bulbs. I have dealt with this subject in the number of the British Journal of Dernuatology for February, 1897. I content myself with drawing attention to these additional diseases and conditions as possible subjects for iscussion.

It has been suggested to me that acne in its varieties, and boils, and carbuncles were diseases allied to cczema. Pustular eczema, impetigo contagiosa, boils, and carbuncles are, at all events, due to the same cause. There are plenty of explanations as to why we should find pustular eczema in one patient, a suppurative folliculitis in another, and acne, boils, or carbuncles in another. The reasons for these various manifestations, if they are due to one actual cause, might form a part of the discussion. In connexion with all these pustular forms of skin diseases I think we may be apt to forget that pus may exist without the presence of pus cocci.

\section{The Treatuent of Eczima.}

With regard to the treatment of eczema one of our members said to me only the ather day that he treated his skin patients with zinc ointment and left the rest to Providence. It is an easy method of disposing of them, but $I$ am sure it will be better for the patients if they are carefully and systematically treated. The physician in ordinary can say much to his patients without any.fear of contradiction, but patients suffering from skin disease can see and note progress for themselves.

The first great principles in the treatment of eczema and its allied diseases are to soothe when the conditions are acute and to carefully stimulate the skin into healthy action as inflammation and irritation subsides. In a diffuse eczema one of the most important soothing elements is rest; oftentimes absolute rest is required.

Where there are a lot of dirt and crusts about all sbould be removed before any special form of treatment be adopted. Thorough soaking in weak carbolic oil is one of the best methods of loosening crusts, whether upon the head or elsewhere. The most careful and explicit directions must be given as to the removal of crusts from the scalp. There are various other substances that may be used for the removal of crusts, for instance, boric starch poultices, solutions of bicarbonate of soda or borax, or fitting the head or limb with an impermeable covering. Having removed all crusts and dirt as far as possible the next thing is to soothe and disinfect. There is hardly a skin disease which during some portion of its career does not require anti-parasitic treatment, and the application requires a great amount of care. It would not do to rush away with the idea that sulphur ointment would always do as the disinfectant. If my good friend aforesaid ever should go outside his zinc ointment treatment for acute eczema and the like diseases I think he would apply sulphur ointment, with the result, perhaps, that he would produce a dermatitis. Not that I am laughing at sulphur, for carefully applied in strengths suitable to each case it is an invaluable remedy. Mercury, ichthyol, tar, resorcin, and similar parasiticide remedies suit still better, some in one case, some in another. Experience gradually teaches us when to order lotions or sprays and when ointments or pastes. In any case the remedy must be kept at work. It is not a bit of good simply perfunctorily to tell the patient to dab on this or that ointment or lotion; he must be told how to apply it and how often to apply it.

It would be interesting to hear what members think with regard to the washing of eczema patients. I think that the patients should be washed from time to time for the better disinfecting of the parts, but I should in all cases order soft water or softened water and careful drying afterwards, and the general directions given for the cleansing of the patient should be similar to those that would be given for the cleansing of a wound. Lassar is one of the strongest adrocates for these periodic washings, but many dermatologists are much against water being used to the skin in eczematous conditions. This is a fair question for discussion. Lassar particularly recommends tar-baths.

There is one caution about washes and sprays that everyone might not think of, and that is if the skin is broken do not order spirituous sprays or washes. Ordinary frequen washings in eczema and dermatitis are not to be allowed. The cleansing and disinfecting of the parts is the point. For macular and erythematous eczema, and also for some forms of weeping eczema, personally I think very highly of dusting powders. French chalk, cimolite, and powdered talc all make useful dusting powders. Oxide of zinc or starch powder is good for astringing or stiffening purposes, and salicylic acid, boric acid, or sulphur may be added for disinfecting purposes. I have used muslin bags filled with powder, as suggested by Unna, with excellent effect in sitna. tions like the groin.

Dry patches of chronic eczema, either bypertrophic or atropbic, are amongst the most troublesome conditions to cure; so, too, are those bypertrophic patches of lichen planus that one so frequently meets with. Stimulation, often strong stimulation, is necessary, but the results must be watched. It seems to me that what suits one case does not suit another and it is often necessary to ring the changes. Strong mercurial or chrysarobin ointments may be useful, tar in some form, or a salicylic and creasote paste, or they may be painted with 10 per cent. to 20 per cent. solutions of caustic potash or nitrate of silver. Blistering is also a valuable means of starting a cure. Of these and of the many other remedies the secret of success with all of them is to keep them at work. Of course, this does not apply to blistering or to the application of strong caustic solutions. Probably some members will give their experience of the gelatin preparations and other varnishes and pastes now so largely used. I am very fond of a medicated plaster in some cases of chronic eczema; I think their great drawback is the fact that they are probably not recently made.

I have said nothing about baths, but having shown myself on the favourable side for the cleansing of eczematous surfaces I have practically committed myself to batbs and I fancy there are but few who would not sometimes order bath in skin diseases. Baths of soda and borax, and glutinous, starch, and bran baths we must frequently order; of the rest sulphur and tar baths are perhaps the best. I think the value of baths and their methods of application would be an excellent point in connexion with treatment for discussion.

Vith regard to the general treatment of eczema and the allied diseases $I$ attach less value to this than to the local treatment. Nevertheless, in conjunction with local applica tions it must often be of service. In acute diseaseespecially in plethoric and gouty subjects-undoubtedly free purgation and the use of drugs that will lessen vaso-motor resistance and produce a free action of the skin, kidneys and mucous tracts generally will be of service. In diabetic skins, whether the condition be xanthoma or ordinary eczematous irritation or dermatitis, undoubtedly general treatment must be gone into. Also where there is evidence of trophic disturbance the nerve change or nerve instability has to be corrected if it be possible to do so. Neurosis is given great importance to by some dermatolcgists, and sedatives, such as the bromides, and stimulants or restoratives, such as the zinc salts or arsenic or strychnine, sbould be serviceable. Personally, I think that when we have to deal with such a class of drugs for the cure that we are getting into troubled waters.

Diet in some cases is of importance, but I think time would hardly permit the discussion of the dietary treatment It is practically treating acute or chronic indigestion. With regard to the treatment at health resorts, and particularly at bathing places and spas generally, no doubt many patients will be benefited by visits to Harrogate, Buxton, Strathpeffer, Aix-les-Bains, Carlsbad, Homburg, and the like places, but it is usually, I think, the patient's general condition rather than the particular form of skin disease that suggests the particular resort. The seaside is not generally suitable for acute or irritable skin disease, but such a statement requires modification.

I do not know why, but it seems to me that medical men as a rule do not trouble themselves much about skin diseases; indeed, they are either to be cured with a dose of white mixture and some zinc ointment or there is nothing to be done for them, and yet I would venture to say that the skin with all its glands and appendages and cells is as beautiful and intricate an organ as any we have to deal with. Its duties in the life-history of the individual are manifold and wonderful to think upon. The physiology of the corium and epidermis and of the appendages which strike down into the subcutaneous tissues teaches us more than a little of the way we live and move and have our being. Its pathology is equally instructive and beautiful; bacteriology probably plaps a more important part for it than it does for any other class 
of disease. Without this knowledge how can we successfully apply our treatment? Yet how few of us take the trouble to master it and what is the result? If we choose to neglect what is really a beautiful and sightly organ when it is sound the patient with an unsound skin tries to get it cured elsewhere. He dodges here and there, tries first one quack nostrum and then another, usually goes from bad to worse from want of systematic guidance, and occasionally stumbles upon a right remedy at the right time and finds himself better or cared. Forthwith he curses the whole priesthood of medicine and extols the quack nostrum to the skies and the quack proprietor shouts it beyond such trivial confines and space will not hold his jubilations. Of the 101 not cured one hears nothing, even less than nothing, if they return for help to the proper channels. Here some of them will find a kindly and sympathetic practitioner who will take the necessary systematic trouble to alleviate and cure them. However, he is not the man to holloa and the patient soon forgets that he has been ill.

I feel that I ought to apologise for trespassing so much on this one line of argument, but I do it because I feel that if we are to beat down quackery and quack nostrums it is to be done by stronger personal efforts on our part to face troublesome and nasty cases instead of just throwing them over as nuisances. All this applies with more force to the difficulties of dermatology than to any other groups of disease. I believe that the profession is really more in the dark abont the knowledge of and the treatment of skin diseases than of any other disorders, perhaps because we have thought that we saw everything and that to know about it and treat it was so very easy. We saw when we had failed and desisted in disgust; the physician in ordinary seldom knows when he has failed and plods on merrily with bispatient perhaps as happy as himself. Hard workers are lifting the reil that has prevented the medical man from always producing good results in eczema and the allied diseases, and I will end by quoting an old negro proverb that has just come into my head- "It takes heaps o' licks to dribe a nail in de dark."

Reading.

\section{A CASE OF PUERPERAL SEPTICEMIA} TREATED BY ANTI-STREPTOCOCCIC SERUM. AND COMPLICATED BY PHLEGMASIA; RECOVERY.

\section{By A. HAMiltoN WoOD, II.D., Ch.B. Edis.}

THE patient whose illness I am about to discuss was confined on the afternoon of May 5th. The first stage was a long and tedious one, due mainly to extreme rigidity of the perineum. Tnere was a superficial tear which, though very slight, may undoubtedly have been the source through which septic matter gained entrance into the system, seeing that the house and its occupants were by no means clean. I visited the patient on the following day and found her perfectly comfortable. She had little or no after-pains. I caw her again on the next day and found her much the same as before; the temperature was normal but the pulse was a little rapid. I was then rather afraid of some septic mischief setting in, and on calling on the following day, which was the third day after her confinement, I found her temperature to be $101^{\circ} \mathrm{F}^{\prime}$. and her pulse 120 . I gave her then five-grain doses of quinine with some hydrobromic acid to prevent as far as possible the uncomfortable head symptoms which are so apt to follow the continued use of this drug; also 15-minim doses of digitalis. She had some castor oil which moved the bowels well but had no effect on the temperature. I sent for one of the district nurses who douched her night and morning with 1 in 1000 corrosive (intra-uterine). The next day the temperature ran up to $104^{\circ}$ and on the same night it was $105^{\circ} 6^{\circ}$. She complained of no pain and told me that she felt "all right but for a slight headache and a dreadful thirst." She was very sleepy and her expression was not anxious but rather drowsy and apathetic. The uterus, though enlarged and contracting badly, was not very tender, but there was a copious foul discharge, a lot of shreddy material returning each time with the douche. On this day, the fourth after confinement, I injected 10 cubic centimetres of anti-streptococcic serum, ordered her a little brandy every three hours, milk and soda-water, bovril, fce, and when I called the next day I found that the temperature had fallen from $105^{\circ} 6^{\circ}$ to $102 \cdot 6^{\circ}$. On that evening the temperature was about the same $102 \cdot 8^{\circ}$, so I did not inject any more serum. On the following morning it had fallen still further to $10 \mathrm{I}^{\prime} 6^{\circ}$, but rose again in the evening to $1042^{\circ}$. This was on May 11th, the sixth day after confinement. In the evening I again injected 10 cubic centimetres of the serum, the chart showing a fall on the next morning to $101^{\circ}$, but in the evening it again rose to $1042^{\circ}$. Oar supply of serum having unfortunately come to an end I could not inject any on that day. On May 13th the temperature in the morning was $100.4^{\circ}$ and in the evening $104^{\circ}$, so I again injected 10 cubic centimetres of the serum. On the next day-that was about a week after the onset-the temperature fell to $100.6^{\circ}$; in the evening it was $101^{\circ}$. I now stopped the serum injections bat continued the quinine and intrauterine douching. The pulse, which up to now had varied with the temperature, had also fallen and was' steadying down. I may mention en passant that from the commencement the secretion of milk was entirely arrested. On the 15th the temperature was $100.6^{\circ}$ in the morning and $102^{\circ}$ in the evening, but as the pulse was steady I did not mind the slight rise. On the next day it was a little above $100^{\circ}$ and the patient was feeling a great deal better. The temperature fell still further on the following day and on the 18th it was down to normal, the pulse-rate falling simultaneously. The discharge, which for the last five or six days had been gradually diminishing, was now very slight. On the next day, however, the patient complained of a little pain down the back of the left leg, and on examining it I found the leg slightly swollen and tender, more especially posteriorly. There was not much tenderness above the popliteal space. The temperature ran up to $99.6^{\circ}$ and the pulse to 86 . For two days before this phlebitis set in; the bowels had not been opened. The patient had taken some castor oil, but bad failed to get relief till an enema was giren. I swathed the leg in cotton.wool and raised the foot of the bed. The quinine and digitalis were continued with the addition of some morphia to allay the pain. For the next two days the temperature remained at about $100^{\circ}$ and on the following day she complained of pain in her right leg; this was only slightly swollen and not so tender as the left. The leg was wrapped in cotton-wool and banclaged as before. It is interesting to note that the lef leg, as is invariably the case, was the first to be attacked and the severer of the troo. On the following day the temperature fell to normal and remained so, except for a slight rise one erening due possibly to constipation. Towards the end of the month all pain had left the legs and by a little gentle massage the remaining swelling quickly disappeared. Since June 1st the patient has been getting up for a little each day, and at the time of writing (June 4th) she says that she feels herself again.

This case is deser'ing of some note, more especially with regard to the mode of treatment adopted. On the sixth day after the commencement of the disease, during which time three injections of serum had been given, the temperature fell considerably and remained down. But the question arises, would the same state of affairs have resulted from the administration of the quinine and digitalis alone without the anti-streptococcic injections? I think not. The intra-uterine douching, no doubt, played an important part in the treatment of the case, but apart from this I am of opinion that the success of the case was to a great extent due to the use of the anti-streptococcic serum. With regard to the aftereffects, let me remark, the patient had absolutely no discomfort. Some hold that the serum produces a depressant action on the heart. It may be that three doses of 10 cubic centimetres each is not sufficient to cause any depression; all I can say is that in this case there was no depression, nor were there any uncomfortable symptoms whatever, either after each dose or after the third had been given.

Then a word or two with regard to the phlegmasia. It started in the left leg and a few days afterwards the right was affected, but to a less degree than the left. For a day or two previously she har been very constipated. This may be a mere coincidence, but it seems to support the theory that the rectum, lying as it does on the left side, has something to do with the phlebitis invariably starting on that side. This case, as well as others already published, may aid in inciting the profession to the further use of 